\title{
Matching more than three moments with acyclic phase type distributions
}

\author{
András Horváth ${ }^{1}$, Miklós Telek ${ }^{2}$ \\ ${ }^{1}$ Dip. di Informatica, Università di Torino \\ ${ }^{2}$ Dept. of Telecommunications, Technical University of Budapest \\ E-mail: horvath@di.unito.it, telek@hit.bme.hu
}

May 17, 2006

\begin{abstract}
This paper presents a matching procedure for generating an acyclic phase type distribution of order $N$ given the first $2 N-1$ moments, if they are feasible. The matching procedure uses an iterative approach and, theoretically, it can be applied to match an arbitrary number of moments. The first step of the iterative procedure contains the solution of an equation of order $N$ and the order is decreased by one in each consecutive step. Apart of these equations the procedure makes use of explicit expressions. The practical applicability of the proposed procedure is limited by the numerical accuracy of the solution of these equations and the complexity of the involved expressions. We present examples for matching more than 10 moments with acyclic phase type distributions.
\end{abstract}

Keywords: acyclic phase type distribution, moments matching.

\section{Introduction}

The applicability of phase type distributions in stochastic models of real systems is determined by the availability of appropriate phase type fitting procedures ${ }^{1}$. There are two main categories of phase type fitting algorithms: numerical optimization $[1,5]$ and procedures that make use of explicit expressions. Up

\footnotetext{
${ }^{1} \mathrm{~A}$ survey of fitting algorithms can be found in [6].
} 
to now explicit expressions for matching only the first three moments of acyclic phase type distributions (APH) have been known $[9,7,8,2]$. This paper presents an iterative approach to match an arbitrary number of moments with acyclic phase type distributions.

The solution of matching an arbitrary number of moments with matrix exponential distributions, which is an even richer class of distributions, containing the APH class, has been known for more than a decade, see [10]. However, the applicability of this matching procedure is limited because the class of matrix exponential distributions and the properties of the matching method have the following disadvantages.

- The matrix exponential class contains the phase type and the acyclic phase type classes, but it also contains distributions which cannot be represented as the time to absorption in a Markov chain. These non-Markovian distributions might inhibit the use of the widely applied Markovian solution techniques.

- The matching procedure calculates a matrix for any set of moments without indicating if the matrix (together with the predefined initial and final vectors) corresponds to a matrix exponential distribution or not. When the procedure is called with the moments of a matrix exponential distribution of order $N$ it results in a true matrix exponential distribution of order $N$, but when it is called with an invalid set of moments it results in a matrix which does not correspond to a real distribution. Unfortunately, it is hard to check if a given matrix corresponds to a real matrix exponential distribution [4].

The analytical description of the moment bounds of matrix exponential distributions would avoid the second disadvantage, but they are not known beyond $N=2$. The procedure presented in this paper avoids the above mentioned disadvantages. APH matching results in a Markovian representation by definition and the feasibility of the solution is obvious (a solution with real, positive intensities and real probabilities between 0 and 1 is feasible).

With the procedure presented in this paper, matching the moments of an acyclic phase type distribution of order $N(\operatorname{APH}(N))$ requires the solution of an equation of order $N$. As a consequence, the matching procedure has a symbolic solution up to $N=4$ and numerical techniques are applicable for larger $N$. Here we present the symbolic solution for $N=3$ (matching 5 moments). Because of the complexity of the symbolic solution for $N=4$ we use a numerical technique for $N \geq 4$. The practical applicability of the proposed procedure is limited by the complexity of the involved expressions and by the numerical 
accuracy of the solution of the equation of order $N$. We present numerical results for matching more than 10 moments.

\section{Notations and theoretical background}

\section{$2.1 \quad$ Normalized moments}

Based on the ordinary moments $m_{i}$ we define the $i$ th normalized moment as follows:

$$
n_{i}=\frac{m_{i}}{m_{i-1} m_{1}}, \quad i \geq 1
$$

Note that throughout this paper we consider only non-defective distributions whose 0th moment is assumed to be 1 . As a consequence the first normalized moment of the considered distributions is 1 (i.e., $m_{0}=1$ and $\left.n_{1}=1\right)$.

We introduce the normalized moments for two reasons.

- They simplify the problem of matching. With the use of normalized moments one can match the first moment and the normalized moments, $n_{i}, 2 \leq i \leq N-1$, separately.

- They simplify the obtained expressions. E.g., the $i$ th normalized moment of an $\operatorname{Erlang}(N)$ distribution is $\frac{N+i-1}{N}$.

Based on (1), the ordinary moments can be calculated from the normalized moments using

$$
m_{i}=n_{i} m_{i-1} m_{1}=m_{1}^{i} \prod_{k=1}^{i} n_{k}
$$

\subsection{Acyclic phase type distributions}

Definition 1. An acyclic phase type distribution of order $N$, denoted by $A P H(N)$, is the distribution of the time to absorption in an acyclic Markov chain with $N$ transient states and an absorbing one.

Theorem 2. [3] Any $A P H(N)$ distribution can be represented in the canonical form (CF) depicted in Figure 1 such that $\lambda_{1} \leq \lambda_{2} \leq \ldots \leq \lambda_{N}$.

Definition 3. The representation of an $A P H(N)$ in the structure of Figure 1 without any restrictions on the order of the intensities is called its series representation. 


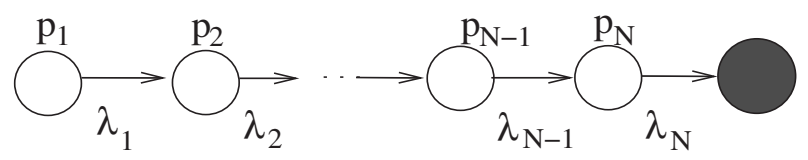

Figure 1: Canonical representation of $\operatorname{APH}(N)$ distributions

Both, the series and the canonical representations of an $\operatorname{APH}(N)$ distribution are defined by two vectors $\underline{p}=\left\{p_{1}, \ldots, p_{N}\right\}$ and $\underline{\lambda}=\left\{\lambda_{1}, \ldots, \lambda_{N}\right\}$.

Corollary 4. The $\underline{\lambda}$ vectors of the different series representations of an $A P H(N)$ contains the same intensities. They differ only in the order of these intensities.

Proof. The corollary is a consequence of Cumani's result [3], but it can be derived also from the fact that the eigenvalues of any triangular matrix are determined by the diagonal elements.

\subsection{The $2 N$ th and the $2 N+1$ th normalized moments of $\operatorname{APH}(N)$ distributions}

As shown by Cumani in [3] an $\operatorname{APH}(N)$ distribution is characterized by $2 N-1$ parameters. According to [10], the first $2 N-1$ moments of an $\operatorname{APH}(N)$ characterize the distribution ${ }^{2}$. As a consequence, one can calculate the higher moments of an $\operatorname{APH}(N)$ in terms of the first $2 N-1$ moments based on [10] (see Appendix A). It is straightforward to transform this result into the relation of the normalized moments

$$
n_{2 N}=\mathcal{F}_{2 N}\left(n_{2}, \ldots, n_{2 N-1}\right), \quad n_{2 N+1}=\mathcal{F}_{2 N+1}\left(n_{2}, \ldots, n_{2 N}\right)
$$

For $N=2$ the second and third normalized moments determine all higher normalized moments. In this case the fourth and fifth normalized moments are

$$
\begin{gathered}
n_{4}=\frac{2 n_{2}\left(9 n_{2}+2\left(n_{3}-6\right) n_{3}\right)}{3\left(n_{2}-2\right) n_{3}}, \\
n_{5}=\frac{5 n_{3}\left(4 n_{2}\left(2 n_{3}-3 n_{4}\right)+3 n_{4}^{2}\right)}{6\left(2 n_{3}-3 n_{2}\right) n_{4}} .
\end{gathered}
$$

\footnotetext{
${ }^{2}$ Indeed [10] proves this statement for the whole matrix exponential class using reduced moments.
} 
For $N=3, n_{2}, n_{3}, n_{4}$ and $n_{5}$ determine all higher normalized moments. In this case

$$
\begin{gathered}
n_{6}=\left(n_{3}\left(25 n_{2}\left(2 n_{2}\left(16 n_{3}^{2}-36 n_{3} n_{4}+9 n_{4}^{2}\right)-3 n_{3}\left(n_{4}-8\right) n_{4}^{2}\right)\right)+\right. \\
\left.n_{3}\left(120 n_{2}\left(6 n_{2}+n_{3}\left(n_{4}-4\right)-3 n_{4}\right) n_{4} n_{5}-36\left(n_{2}-2\right) n_{4}^{2} n_{5}^{2}\right)\right) / \\
\left(10 n_{4}\left(18 n_{2}^{2}+6 n_{3} n_{4}+n_{2} n_{3}\left(4 n_{3}-3\left(8+n_{4}\right)\right)\right) n_{5}\right) \\
n_{7}=\left(7 n _ { 4 } \left(-8 n_{3} n_{5}^{2}\left(3 n_{4}\left(3 n_{5}-5 n_{6}\right)+5 n_{6}^{2}\right)+5 n_{2}\left(12 n_{5}^{2} n_{6}^{2}+12 n_{3} n_{5}\left(6 n_{4} n_{5}-5 n_{4} n_{6}-4 n_{5} n_{6}\right)+\right.\right.\right. \\
\left.\left.\left.n_{3}^{2}\left(75 n_{4}^{2}-180 n_{4} n_{5}+48 n_{5}^{2}+80 n_{5} n_{6}\right)\right)\right)\right) /\left(2 0 n _ { 5 } \left(5 n_{3}\left(4 n_{2}\left(2 n_{3}-3 n_{4}\right)+3 n_{4}^{2}\right)+\right.\right. \\
\left.\left.6\left(3 n_{2}-2 n_{3}\right) n_{4} n_{5}\right) n_{6}\right) .
\end{gathered}
$$

Because of their complexity, we do not provide the expressions for higher order moments. These expressions can be calculated up to $N=8$ in about two minutes with a standard personal computer (1.5 GHz processor and 524MB RAM). The computation for $N=9$ has such high memory requirement that it cannot be performed with a computer with 524MB RAM.

\section{The matching procedure}

It is straightforward to compute the normalized moments, $n_{i}, 2 \leq i \leq 2 N-1$, of an $\operatorname{APH}(N)$ distribution in terms of its $2 N-1$ parameters. The inverse, i.e. to determine the parameters given the normalized moments, is non-trivial. In this section we present an iterative approach which, at each step, determines two parameters of the $\operatorname{APH}(N)$ distribution.

The section starts with the building blocks and then describes the procedure itself. Section 3.1 investigates the effects of extending an $\operatorname{APH}(N-1)$ distribution with an additional phase. Based on properties of the extension, the problem of matching $2 N-2$ normalized moments with an $\operatorname{APH}(N)$ distribution can be reduced to the problem of matching $2 N-4$ normalized moments with an $\operatorname{APH}(N-1)$ distribution. This reduction step is described in Section 3.2. The complete algorithm is presented in Section 3.4. 


\subsection{The $\operatorname{APH}(N-1)$-EXP structure}

The extension of an $\operatorname{APH}(N-1)$ distribution with an additional phase, as depicted in Figure 2, is characterized by two parameters, $p$ and $\lambda$. The following theorem and its corollary describe the effect of the extension on the moments and normalized moments, respectively.

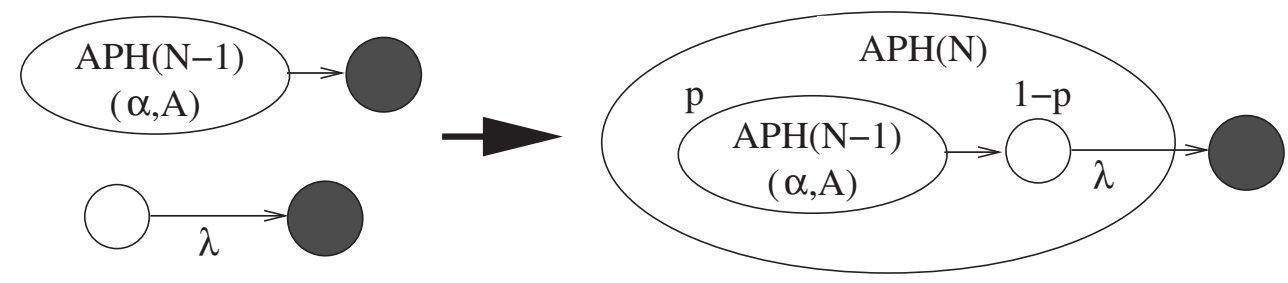

Figure 2: Realization of an $\operatorname{APH}(N)$ distribution by the composition of an $\operatorname{APH}(N-1)$ distribution and an additional phase

Theorem 5. The moments of the resulting APH(N-1)-EXP distribution, $m_{i}^{\prime}$, can be expressed in terms of the moments of the $A P H(N-1)$ distribution, $m_{i}$, and the two parameters of the extension ( $p$ and $\left.\lambda\right)$, using

$$
m_{i}^{\prime}=i ! \lambda^{-i}\left(1+p \sum_{j=1}^{i} \frac{\lambda^{j} m_{j}}{j !}\right)
$$

Proof. Let $f_{L}(s)$ be the Laplace transform of the $\operatorname{APH}(N-1)$ distribution. The Laplace transform of the $\operatorname{APH}(N-1)$-EXP distribution, $g_{L}(s)$, is

$$
g_{L}(s)=p f_{L}(s) \frac{\lambda}{s+\lambda}+(1-p) \frac{\lambda}{s+\lambda}=\frac{\lambda}{s+\lambda}\left(p f_{L}(s)+1-p\right)
$$

To obtain its moments we first take the $i$ th derivative of (9)

$$
\begin{aligned}
& \frac{d^{i}}{d s^{i}} g_{L}(s)=\sum_{j=0}^{i}\left(\begin{array}{l}
i \\
j
\end{array}\right) \frac{d^{i-j}}{d s^{i-j}}\left(\frac{\lambda}{s+\lambda}\right) \frac{d^{j}}{d s^{j}}\left(p f_{L}(s)+1-p\right)= \\
& \sum_{j=1}^{i}\left(\begin{array}{l}
i \\
j
\end{array}\right) \lambda(i-j) !(-1)^{i-j}(s+\lambda)^{-i+j-1} p \frac{d^{j}}{d s^{j}} f_{L}(s)+\lambda i !(-1)^{i}(s+\lambda)^{-i-1}\left(p f_{L}(s)+1-p\right)
\end{aligned}
$$

then by the limit $s \rightarrow 0$

$$
(-1)^{i} m_{i}^{\prime}=\sum_{j=1}^{i}\left(\begin{array}{l}
i \\
j
\end{array}\right)(i-j) !(-1)^{i-j} \lambda^{-i+j} p(-1)^{j} m_{j}+i !(-1)^{i} \lambda^{-i}
$$


Multiplying both sides by $(-1)^{i}$ gives (8).

To simplify the following expressions and to eliminate the dependence on the first moment, we replace the parameters, $p$ and $\lambda$, by

$$
a=m_{1} \lambda \quad \text { and } \quad b=a p .
$$

Indeed $a=\frac{m_{1}}{1 / \lambda}$ is the ratio of the means of the $\operatorname{APH}(N-1)$ and the EXP parts of the $\operatorname{APH}(N-1)-\mathrm{EXP}$ distribution.

Corollary 6. The normalized moments of the APH( $N-1)$-EXP distribution, $n_{i}^{\prime}$, can be expressed in terms of the normalized moments of the APH(N-1) distribution, $n_{i}$, using

$$
n_{i}^{\prime}=\frac{i\left(1+b \sum_{j=1}^{i} \frac{a^{j-1}}{j !} \prod_{k=1}^{j} n_{k}\right)}{(1+b)\left(1+b \sum_{j=1}^{i-1} \frac{a^{j-1}}{j !} \prod_{k=1}^{j} n_{k}\right)}
$$

Proof. From the definition of the normalized moments, using (8) and (2) we have

$$
n_{i}^{\prime}=\frac{m_{i}^{\prime}}{m_{i-1}^{\prime} m_{1}^{\prime}}=\frac{i ! \lambda^{-i}\left(1+p \sum_{j=1}^{i} \frac{\lambda^{j}}{j !} \prod_{k=1}^{j} n_{k} m_{1}^{j}\right)}{\left((i-1) ! \lambda^{-i+1}\left(1+p \sum_{j=1}^{i-1} \frac{\lambda^{j}}{j !} \prod_{k=1}^{j} n_{k} m_{1}^{j}\right)\right)\left(p m_{1}+\lambda^{-1}\right)} .
$$

Introducing the $a$ and $b$ parameters results in (11).

\subsection{Reduction to one phase less}

Given $2 N-2$ normalized moments, $n_{i}^{\prime}, 2 \leq i \leq 2 N-1$, we aim to find $n_{i}, 2 \leq i \leq 2 N-3$, and $a$ and $b$ such that the extension of an $\operatorname{APH}(N-1)$ distribution whose normalized moments are $n_{i}$ with an exponential phase according to Figure 2 with parameters $a$ and $b$, results in an $\operatorname{APH}(N)$ distribution with normalized moments $n_{i}^{\prime}$. To this end we derive the following two corollaries of Theorem 5 .

Corollary 7. The inverse function of (8), i.e., the moments of the APH(N-1) distribution as a function 
of the moments of the APH(N-1)-EXP distribution, have the following from

$$
m_{i}=\frac{\lambda m_{i}^{\prime}-i m_{i-1}^{\prime}}{\lambda p}
$$

Proof. We prove the corollary by induction. (12) holds for $i=1$. Assuming that (12) holds for $j \in$ $\{1,2, \ldots, i-1\}$ from $(8)$ we have

$$
\frac{\lambda^{i}}{i !} m_{i}^{\prime}=1+p \sum_{j=1}^{i-1} \frac{\lambda^{j}}{j !} m_{j}+p \frac{\lambda^{i}}{i !} m_{i}=1+p \sum_{j=1}^{i-1} \frac{\lambda^{j}}{j !} \frac{\lambda m_{j}^{\prime}-j m_{j-1}^{\prime}}{\lambda p}+p \frac{\lambda^{i}}{i !} m_{i}
$$

That is

$$
\frac{\lambda^{i}}{i !} m_{i}^{\prime}=\underbrace{1+\sum_{j=1}^{i-1}\left(\frac{\lambda^{j} m_{j}^{\prime}}{j !}-\frac{\lambda^{j-1} m_{j-1}^{\prime}}{(j-1) !}\right)}_{\frac{\lambda^{i-1} m_{i-1}^{\prime}}{(i-1) !}}+p \frac{\lambda^{i}}{i !} m_{i}
$$

which gives (12) after some manipulation.

Corollary 8. The normalized moments of the $A P H(N-1)$ distribution as a function of the normalized moments of the APH(N-1)-EXP distribution, have the following from

$$
n_{i}=\frac{n_{i-1}^{\prime}(1+b)\left(n_{i}^{\prime}(1+b)-i\right)}{a\left(n_{i-1}^{\prime}(1+b)-(i-1)\right)}, \quad i>1
$$

Proof. From the definition of the normalized moments and (12) we have

$$
\begin{aligned}
n_{i} & =\frac{m_{i}}{m_{i-1} m_{1}}=\frac{\frac{\lambda m_{i}^{\prime}-i m_{i-1}^{\prime}}{\lambda p}}{\frac{\lambda m_{i-1}^{\prime}-(i-1) m_{i-2}^{\prime}}{\lambda p} \frac{a}{\lambda}} \\
& =\frac{\left(\lambda m_{i}^{\prime}-i m_{i-1}^{\prime}\right) \lambda}{\left(\lambda m_{i-1}^{\prime}-(i-1) m_{i-2}^{\prime}\right) a}=\frac{\left(\lambda \frac{m_{i}^{\prime}}{m_{i-1}^{\prime}}-i\right) \frac{m_{i-1}^{\prime}}{m_{i-2}^{\prime}} \lambda}{\left(\lambda \frac{m_{i-1}^{\prime}}{m_{i-2}^{\prime}}-(i-1)\right) a} \\
& =\frac{\left(\lambda n_{i}^{\prime} \frac{1+b}{\lambda}-i\right) n_{i-1}^{\prime} \frac{1+b}{\lambda} \lambda}{\left(\lambda n_{i-1}^{\prime} \frac{1+b}{\lambda}-(i-1)\right) a}
\end{aligned}
$$


where we applied $m_{1}^{\prime}=(1+b) / \lambda$.

Based on (15), the $n_{i}, 2 \leq i \leq 2 N-3$, normalized moments of the $\operatorname{APH}(N-1)$ distribution are explicitly known as a function of $n_{i}^{\prime}, 2 \leq i \leq 2 N-3$, and $a$ and $b$. The two other unknowns, $a$ and $b$, can be determined based on the remaining two normalized moments $n_{2 N-2}^{\prime}$ and $n_{2 N-1}^{\prime}$. An equation to determine $a$ is constructed by the following steps:

Step 1 take (11) for $i=2 N-2$,

Step 2 substitute $n_{2 N-2}$ by $\mathcal{F}_{2 N-2}\left(n_{2}, \ldots, n_{2 N-3}\right)$ as provided by [10],

Step 3 substitute $n_{i}, 2 \leq i \leq 2 N-3$ applying (15).

The resulting equation has two unknowns, $a$ and $b$, and enjoys the following property.

Theorem 9. The equation to determine $a$ is linear in a and hence it can be rearranged to have an explicit expression for a as a function of $n_{i}^{\prime}, 2 \leq i \leq 2 N-2$, and $b$.

In a similar manner, an equation to determine $b$ is constructed by the following steps:

Step 1 take (11) for $i=2 N-1$,

Step 2 substitute $n_{2 N-1}$ by $\mathcal{F}_{2 N-1}\left(n_{2}, \ldots, n_{2 N-2}\right)$ as provided by [10],

Step 3 substitute $n_{i}, 2 \leq i \leq 2 N-2$ applying (15).

The resulting equation has a single unknown variable, $b$, and enjoys the following property.

Theorem 10. The equation to determine $b$ is of order $N$ in $b$ and hence it can be rearranged to have a polynomial equation of order $N$ to determine $b$.

A consequence of Theorem 10 is that one can look for symbolic solution up to $N=4$ (matching 7 moments). Fitting more than 7 moments requires the use of numerical techniques.

The proofs of Theorem 9 and 10 are in Appendix A. We demonstrate the procedure for obtaining $n_{i}, 2 \leq i \leq 2 N-3$, and $a$ and $b$ given $n_{i}^{\prime}, 2 \leq i \leq 2 N-1$, for $N=3$ in Section 4.

\subsection{Feasibility}

The parameters of the $\operatorname{APH}(N-1)$-EXP structure, $a$ and $b$, are positive real numbers with the following bounds, $0<a<\infty$ and $0<b=a p \leq a$ (since $0<p \leq 1$ ). $p=0$ is not feasible because it results in an exponential distribution, and $a=0$ is not feasible because it implies $m_{1}=0$ or $\lambda=0$. 
Theorem 11. A set of normalized moments $\left\{n_{2}^{\prime}, \ldots, n_{2 N-1}^{\prime}\right\}$ is feasible with an $A P H(N)$ distribution if and only if there exists a solution of the reduction step, $n_{i}, 2 \leq i \leq 2 N-3$, and $a$ and $b$ such that

- $a$ and $b$ are real numbers such that $0<b(=a p) \leq a$,

- the normalized moments $\left\{n_{2}, \ldots, n_{2 N-3}\right\}$ are feasible with an $A P H(N-1)$ distribution.

Proof. The theorem is a consequence of Cumani's seminal result (Theorem 2). Since any $\mathrm{APH}(N)$ can be represented in canonical form, there is an $\operatorname{APH}(N)$ with the given moments if and only if there is an $\operatorname{APH}(N-1)$-EXP structure with the given constraints.

The first condition of the theorem can be checked easily. The second condition has to be checked by repeated applications of the reduction step. In each step the number of moments is decreased by two. The whole procedure is described in the next section.

Theorem 11 has an important consequence. It allows us to check if a given set of moments is inside the moments bounds of the $\operatorname{APH}(N)$ class or not. Indeed, the procedure described hereinafter directly constructs an $\operatorname{APH}(N)$ distribution, if possible. To the best of our knowledge it is the first method to check this property for more than three moments.

Due to the simple stochastic interpretation of the APH distribution, it is easy to check if the result of the procedure is a proper APH distribution. This property is valuable with respect to the matrix exponential case where the matrix representation, calculated from the set of moments [10], does not indicate the feasibility of the solution.

\subsection{Iterative approach of moments matching}

Based on the reduction to one phase less presented in Section 3.2, the following iterative algorithm generates an $\operatorname{APH}(N)$ distributions given the first $2 N-1$ moments.

1. We calculate the normalized moments, $n_{i}, 2 \leq i \leq 2 N-1$, based on the first $2 N-1$ ordinary moments, $m_{i}, 1 \leq i \leq 2 N-1$, according to $(1)$.

2. The reduction step can be applied according to the tree scheme depicted in Figure 3. At level zero we have $n_{i}, 2 \leq i \leq 2 N-1$, i.e., the normalized moments. At the first level the reduction step gives at most $N$ different solutions (Theorems 9 and 10). The reduction step can be applied again to any of these solutions leading to at most $N(N-1)$ nodes at the second level. In order to avoid 
cumbersome notation, indices of the different solutions for $b, a$ and $n_{i}$ are indicated on the top of the nodes. At the last level there are at most $N$ ! nodes.

3. Taking any path from the root to a leaf gives a sequence of values for $b$ and $a$. This sequence can be used to construct an $\operatorname{APH}(N)$ distribution with normalized moments $n_{i}, 2 \leq i \leq 2 N-1$. We start with an $\mathrm{APH}(1)$ distribution with rate 1 and in each step the distribution is extended by one phase. The values for $a$ and $b$ are taken from bottom to top and are applied according to (10). Note that, as it will be illustrated later in this section, not all the paths lead to a proper $\operatorname{APH}(N)$ distribution.

4. Finally, we set the first ordinary moment (the mean) by properly scaling the intensities of the result.

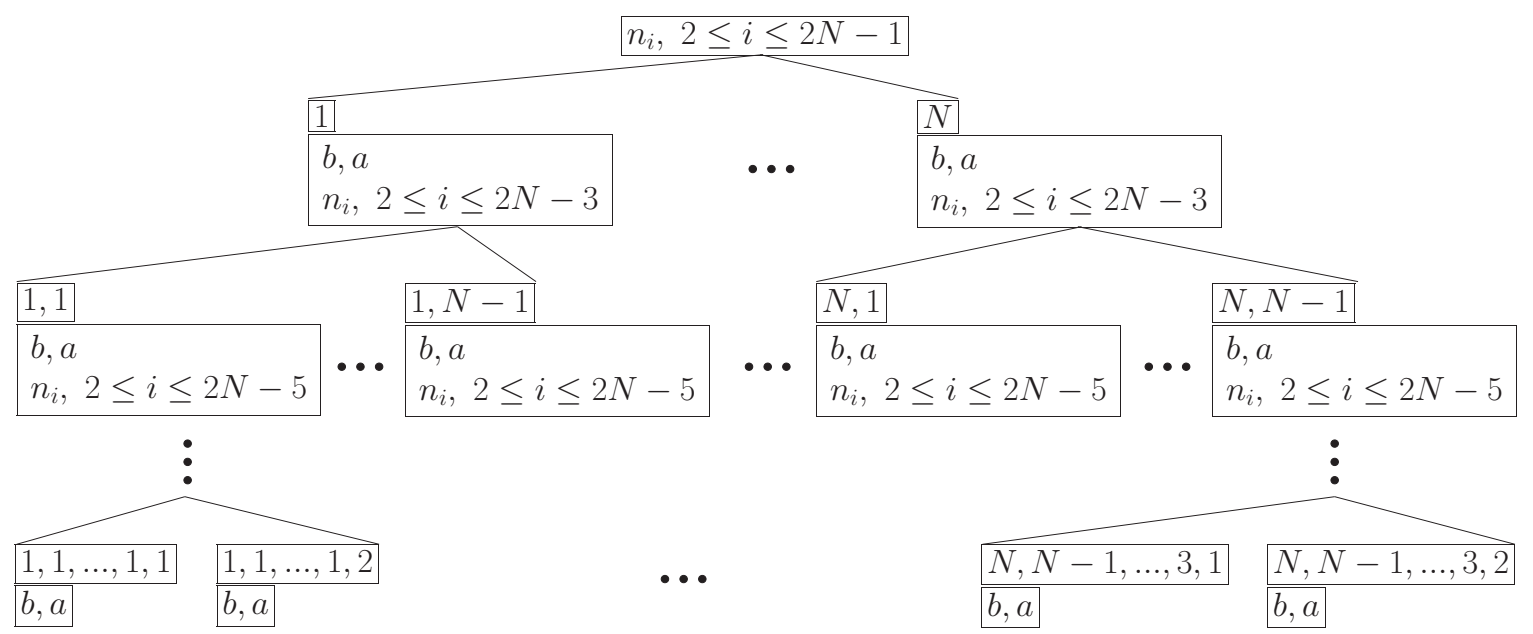

Figure 3: Tree scheme of the application of the reduction step

All the routes provide a series form (with coefficients which are not necessarily feasible). Due to fact that they solve equations (15) and (3), they all provide the given $2 N-1$ ordinary moments.

Corollary 12. The $\underline{\lambda}$ vectors of the $N$ ! solutions contain the same intensity values with all possible permutations.

Proof. The eigenvalues of the $\mathrm{PH}$ generator is determined by the first $2 N-1$ moments of the distribution [10]. In case of APH distributions the eigenvalues are real and they determine the departure rate of the phases. $N$ ! solutions are obtained by the permutations of these eigenvalues.

Let $N=3, m_{1}=1, m_{2}=42 / 25 \cong 1.68, m_{3}=61614 / 15625 \cong 3.94, m_{4}=4654584 / 390625 \cong 11.92$, $m_{5}=17249112 / 390625 \cong 44.16$. The normalized moments are $n_{2}=42 / 25 \cong 1.68, n_{3}=1467 / 625 \cong 2.35$, 


\begin{tabular}{|c|c|c|c|c|}
\hline$a, b$, & $n_{2}, n_{3}$ & $a, b$ & & $\left\{p_{1}, p_{2}, p_{3}\right\},\left\{\lambda_{1}, \lambda_{2}, \lambda_{3}\right\}$ \\
\hline 1 & $\frac{7}{12}, \frac{7}{18}, \frac{100}{49}, \frac{78}{25}$ & 1,1 & $\begin{array}{l}\frac{3}{4},-\frac{1}{8} \\
\frac{4}{3}, \frac{1}{6}\end{array}$ & $\begin{array}{ll}\frac{-1}{9}, \frac{7}{9}, \frac{1}{3} \quad & \frac{25}{9}, \frac{25}{12}, \frac{25}{18} \\
\frac{1}{12}, \frac{7}{12}, \frac{1}{3} & , \frac{25}{12}, \frac{25}{9}, \frac{25}{18}\end{array}$ \\
\hline 2 & $\frac{39}{28}, \frac{13}{12}, \frac{350}{169}, \frac{1029}{325}$ & 2,2 & $\begin{array}{l}\frac{1}{2},-\frac{1}{14} \\
2, \frac{6}{7}\end{array}$ & $\begin{array}{ll}\frac{-1}{9}, \frac{8}{9}, \frac{2}{9} \quad, \quad \frac{25}{9}, \frac{25}{18}, \frac{25}{12} \\
\frac{1}{3}, \frac{4}{9}, \frac{2}{9}, \frac{25}{18}, \frac{25}{9}, \frac{25}{12}\end{array}$ \\
\hline 3 & $\frac{32}{15}, \frac{16}{9}, \frac{125}{64}, \frac{231}{80}$ & 3,2 & $\begin{array}{l}\frac{2}{3}, \frac{1}{15} \\
\frac{3}{2}, \frac{3}{5}\end{array}$ & $\begin{array}{l}\frac{1}{12}, \frac{3}{4}, \frac{1}{6}, \frac{25}{12}, \frac{25}{18}, \frac{25}{9} \\
\frac{1}{3}, \frac{1}{2}, \frac{1}{6}, \frac{25}{18}, \frac{25}{12}, \frac{25}{9}\end{array}$ \\
\hline
\end{tabular}

Table 1: The $N$ ! different solutions of the iterative procedure for $N=3$

$n_{4}=86196 / 28525 \cong 3.02, n_{5}=26619 / 7183 \cong 3.71$. Application of the iterative procedure is summarized in Table 1. The first column gives the three different solutions for $a, b, n_{2}$ and $n_{3}$ at the first application of the reduction step (first level of the tree in Figure 3). Then we apply the reduction step to all of these three solutions. At this level the procedure results in two solutions for $a$ and $b$. These solutions, which are the leaves of the tree depicted in Figure 3, are listed in the second column. In the first two columns the indices of the solutions are indicated as in Figure 3. The corresponding APH(3) distributions are given in the third column by the initial probability vector and the vector of intensities.

As indicated by Corollary 12 the application of the procedure provides all permutations of the same set of intensities. In this particular case 4 permutations of the $3 !=6$ possible permutations result in a proper $\mathrm{APH}(3)$ distribution. These 4 different series representations correspond to the same $\mathrm{APH}(3)$ distribution, whose (unique) canonical representation is in the last row of the table.

The following corollary, whose proof is in Appendix B, indicates how to choose among the different solutions.

Corollary 13. If the given moment set is feasible, selecting the largest solution for $b$ in each step of the 
iterative procedure results in an APH distribution in $C F$, i.e., with non-decreasing intensities. If the given moment set is not feasible, the solutions provided by the procedure are improper for an APH distribution.

Corollary 13 allows us to reduce the $N$ ! possibilities to one. This clearly simplifies the procedure. Furthermore, this solution is in CF which is a minimal unique representation of the APH distributions.

\section{Constructing an $\mathrm{APH}(3)$ with given second to fifth normalized moments}

We apply the iterative procedure for $N=3$ with normalized moments $n_{2}^{\prime}, n_{3}^{\prime}, n_{4}^{\prime}$ and $n_{5}^{\prime}$. In order to construct an equation for $a$, as first step, we take (11) for $i=4$ :

$$
n_{4}^{\prime}=\frac{4\left(1+b\left(1+\frac{1}{2} a n_{2}+\frac{1}{6} a^{2} n_{2} n_{3}+\frac{1}{24} a^{3} n_{2} n_{3} n_{4}\right)\right)}{(1+b)\left(1+b\left(1+\frac{1}{2} a n_{2}+\frac{1}{6} a^{2} n_{2} n_{3}\right)\right)}
$$

Then $n_{4}$ is substituted by $\mathcal{F}_{4}\left(n_{2}, n_{3}\right)$ (given in (4)) which results in

$$
n_{4}^{\prime}=\frac{4\left(1+b\left(1+\frac{1}{2} a n_{2}+\frac{1}{6} a^{2} n_{2} n_{3}+\frac{a^{3} n_{2}^{2}\left(9 n_{2}+2\left(n_{3}-6\right) n_{3}\right)}{36\left(n_{2}-2\right)}\right)\right)}{(1+b)\left(1+b\left(1+\frac{1}{2} a n_{2}+\frac{1}{6} a^{2} n_{2} n_{3}\right)\right)}
$$

By applying (15) to $n_{2}$ and $n_{3}$, and simplifying the right hand side we obtain

$$
\begin{aligned}
n_{4}^{\prime}= & \left(2 \left(9 a\left(-2+n_{2}^{\prime}+b n_{2}^{\prime}\right)\left(4+n_{2}^{\prime}\left(-4+(1+b)^{2} n_{2}^{\prime}\right)\right)-12 a b n_{2}^{\prime}\left(-2-b+(1+b)^{2} n_{2}^{\prime}\right) n_{3}^{\prime}+\right.\right. \\
& \left.\left.\left.2 b(1+b) n_{2}^{\prime}\left(-6 n_{3}^{\prime}+n_{2}^{\prime}\left(9+(1+b) n_{3}^{\prime}\left(-3+n_{3}^{\prime}+b n_{3}^{\prime}\right)\right)\right)\right)\right)\right) / \\
& \left(\left(3 b(1+b) n_{2}^{\prime}\left(-2(1+b+a b)+(1+b)^{2} n_{2}^{\prime}\right) n_{3}^{\prime}\right)\right)
\end{aligned}
$$

from which we have an expression for $a$ as a function of $n_{2}^{\prime}, n_{3}^{\prime}, n_{4}^{\prime}$ and $b$. 
In order to construct the third order equation for $b$ we take (11) for $i=5$ :

$$
n_{5}^{\prime}=\frac{5\left(1+b\left(1+\frac{1}{2} a n_{2}+\frac{1}{6} a^{2} n_{2} n_{3}+\frac{1}{24} a^{3} n_{2} n_{3} n_{4}+\frac{1}{120} a^{4} n_{2} n_{3} n_{4} n_{5}\right)\right)}{(1+b)\left(1+b\left(1+\frac{1}{2} a n_{2}+\frac{1}{6} a^{2} n_{2} n_{3}+\frac{1}{24} a^{3} n_{2} n_{3} n_{4}\right)\right)} .
$$

Then $n_{5}$ is substituted by $\mathcal{F}_{5}\left(n_{2}, n_{3}, n_{4}\right)$ (given in (5)) which results in

$$
n_{5}^{\prime}=\frac{5\left(1+b\left(1+\frac{1}{2} a n_{2}+\frac{1}{6} a^{2} n_{2} n_{3}+\frac{1}{24} a^{3} n_{2} n_{3} n_{4}+\frac{a^{4} n_{2} n_{3}^{2}\left(4 n_{2}\left(2 n_{3}-3 n_{4}\right)+3 n_{4}^{2}\right)}{144\left(2 n_{3}-3 n_{2}\right)}\right)\right)}{(1+b)\left(1+b\left(1+\frac{1}{2} a n_{2}+\frac{1}{6} a^{2} n_{2} n_{3}+\frac{1}{24} a^{3} n_{2} n_{3} n_{4}\right)\right)}
$$

By applying (15) to $n_{2}, n_{3}$ and $n_{4}$, and simplifying the right hand side we have

$$
\begin{aligned}
n_{5}^{\prime}= & \left(\left(5 \left(-72 n_{3}^{\prime} n_{4}^{\prime}+2 n_{2}^{\prime 2}\left(4\left(-3+n_{3}^{\prime}+b n_{3}^{\prime}\right)\left(9+(1+b)^{2} n_{3}^{\prime 2}\right)-3(1+b)^{2} n_{3}^{\prime}\left(-3+2(1+b) n_{3}^{\prime}\right) n_{4}^{\prime}\right)+\right.\right.\right. \\
& \left.\left.\left.3 n_{2}^{\prime} n_{3}^{\prime}\left(96-12 b n_{4}^{\prime}+n_{3}^{\prime}\left(-16(2+b)+4(1+b)(2+b) n_{4}^{\prime}+b(1+b)^{2} n_{4}^{\prime 2}\right)\right)\right)\right)\right) / \\
& \left(\left(6(1+b) n_{3}^{\prime}\left(-12-3(1+b)^{2} n_{2}^{\prime 2}+2 n_{2}^{\prime}\left(6+b\left(3+n_{3}^{\prime}+b n_{3}^{\prime}\right)\right)\right) n_{4}^{\prime}\right)\right)
\end{aligned}
$$

which provides a third order polynomial equation to determine $b$.

Solving (18) and substituting the solutions into (17) and (15) results in three solutions for $n_{2}, n_{3}, a$ and $b$. According to Corollary 13 we can take that with the largest $b$, check the feasibility of $a$ and $b$ and look for an $\operatorname{APH}(2)$ with normalized moments $n_{2}$ and $n_{3}$ by applying the reduction step for $N=2$.

\section{$5 \quad$ Implementation and numerical accuracy}

The presented method is implemented in Mathematica which allows us to check its properties by applying either symbolic calculations (with exact rational representation of the involved quantities) or numerical calculations with different precision. The experiments were carried out on a computer with $1.5 \mathrm{GHz}$ processor and 524MB RAM.

The exact rational arithmetic is applicable only to moment sets that correspond to solution vectors composed of rational numbers. We generated such moment sets by starting from an APH distribution with rational initial probabilities and intensities. Based on the obtained rational moments the procedure 


\begin{tabular}{|c||c|c|c|c|c|c|}
\hline & $n_{2}$ & $n_{3}$ & $n_{4}$ & $n_{5}$ & $\underline{p}$ & $\underline{\lambda}$ \\
\hline \hline D1 & 2.5 & 10 & 34.16 & 52.23 & $2.41210^{-3}, 9.97510^{-1}$ & $9.35210^{-2}, 1.026$ \\
D2 & 2.5 & 10 & 100 & 200 & $3.34910^{-5}, 1.20510^{-1}, 8.79410^{-1}$ & $2.43610^{-2}, 5.38010^{-1}, 1.291$ \\
D3 & 2.5 & 10 & 50 & 200 & $4.38310^{-7}, 2.88410^{-3}, 9.97110^{-1}$ & $1.22810^{-2}, 1.02410^{-1}, 1.029$ \\
D4 & 2.5 & 10 & 100 & 400 & $9.18710^{-7}, 4.95810^{-3}, 9.95010^{-1}$ & $1.04110^{-2}, 1.33510^{-1}, 1.038$ \\
D5 & 2.5 & 10 & 50 & 400 & $9.50010^{-9}, 2.57510^{-3}, 9.97410^{-1}$ & $4.82810^{-3}, 9.65410^{-2}, 1.027$ \\
\hline
\end{tabular}

Table 2: Normalized moments and descriptors of APH distributions for Example 1

calculated exactly the starting APH distribution for 8 phases (matching 15 moments) in 165 seconds.

We found that the standard floating point precision of Mathematica (16 digits) is usually sufficient up to $N=5$, but the round-off error is already visible in the 8 th digit of the normalized moments. Fitting higher order APH distributions requires higher numerical precision. For $N=5$, using 32 digit accuracy floating point arithmetic, the computation time is approximately the same and the round-off error disappears. The computational complexity of matching higher order APH distributions increases exponentially with the order. The calculations for 5,6,7 and 8 phases takes about 2.5, 5.5, 32 and 165 seconds, respectively. For $N>8$ we cannot perform the procedure because with the computer we use we are not able to provide the functions $\mathcal{F}_{2 N}\left(n_{2}, \ldots, n_{2 N-1}\right)$ and $\mathcal{F}_{2 N+1}\left(n_{2}, \ldots, n_{2 N}\right)$.

\section{Examples}

We demonstrate the properties of moments based APH distribution matching through some simple numerical examples.

Example 1: We construct APH distributions with $m_{1}=1$ and the sets of normalized moments listed in Table 2. Distribution D1 is the result of matching normalized moments $n_{2}=2.5$ and $n_{3}=10$ with an $\operatorname{APH}(2)$. All higher order moments of this distribution are determined by $n_{2}$ and $n_{3}$. All other cases are obtained by $\operatorname{APH}(3)$ distributions. Figure 4 presents the body and the tail of the pdf of the distributions. The queue length distribution of the $\mathrm{M} / \mathrm{APH} / 1$ queue with $\rho=0.8$ utilization is provided in Figure 5. Even if the second and third normalized moments are identical, the distributions and the associated 
queue length distributions can differ significantly. In particular, we can recognize the difference between the role of the odd and the even moments. Comparing, for example, D2 and D4 $\left(\left\{n_{2}, n_{3}\right\}=\{100,200\}\right.$ and $\left.\left\{n_{2}, n_{3}\right\}=\{100,400\}\right)$ indicates that a higher fifth moment results in a longer tail for the pdf and also for the queue length distribution. Instead, comparing D2 and D3 $\left(\left\{n_{2}, n_{3}\right\}=\{100,200\}\right.$ and $\left.\left\{n_{2}, n_{3}\right\}=\{50,200\}\right)$ indicates that a higher fourth moment results in a shorter tail.
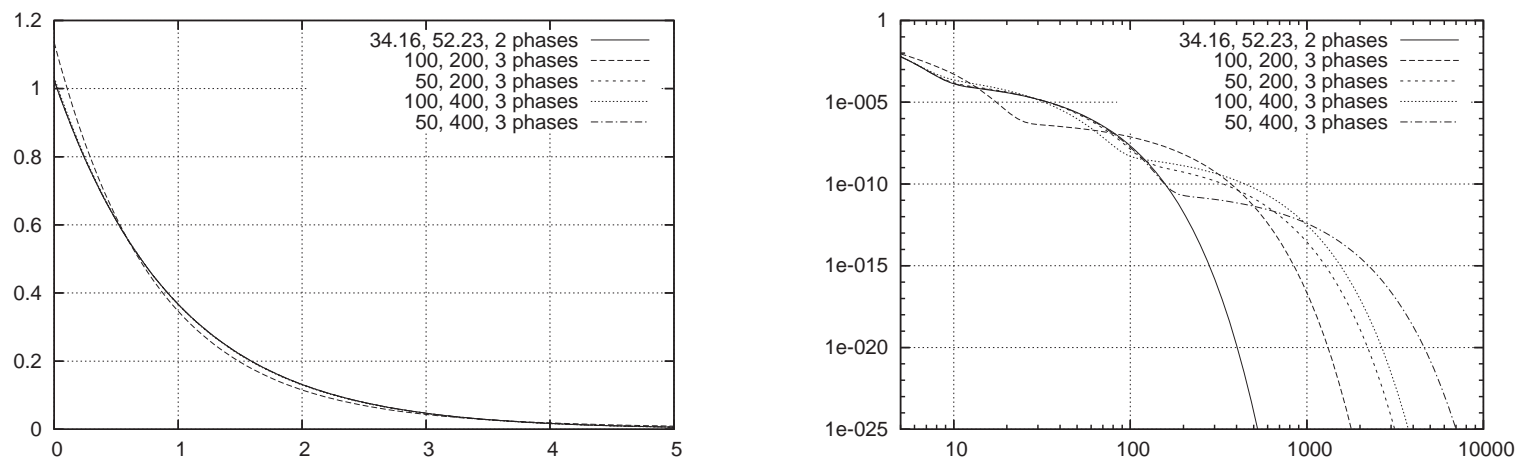

Figure 4: The body and the tail of the pdf of the distributions D1-D5
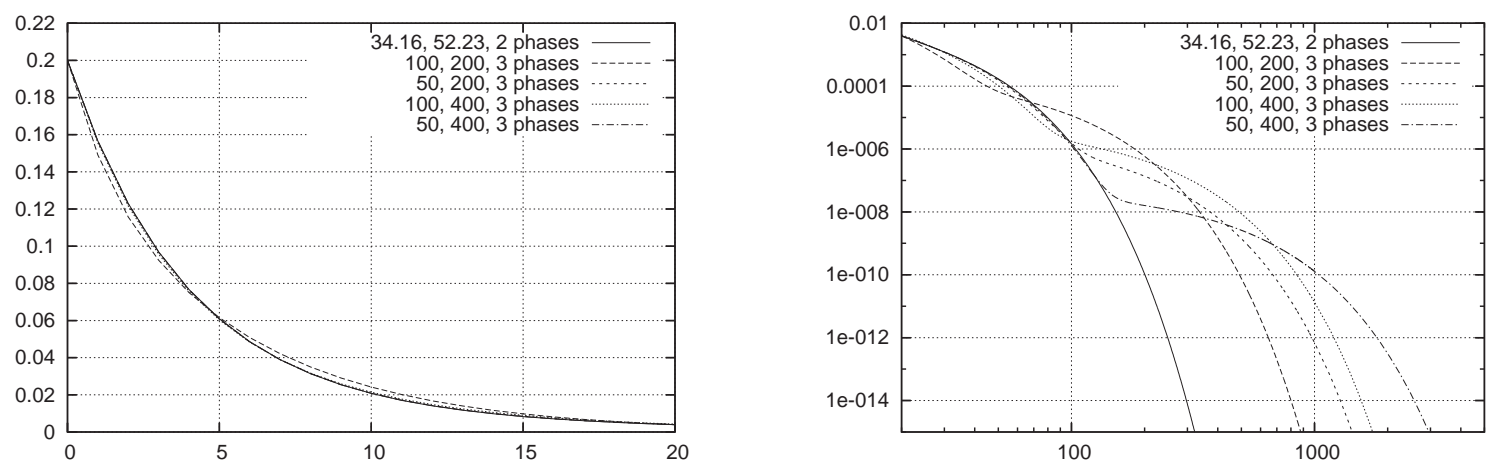

Figure 5: The body and the tail of the queue length distribution of the $\mathrm{M} / \mathrm{APH} / 1$ queue for distributions D1-D5

Example 2: We match an APH distribution with $m_{1}=1$ to sets of normalized moments listed in Table 3. Similar to Example 1, the first set of moments of this example (D6) can be realized with an $\mathrm{APH}(3)$ distribution. The other cases require 4 phases. The pdf and the queue length distribution of the M/APH/1 queue with $\rho=0.8$ utilization are provided in Figures 6 and 7, respectively. The different behaviour of the odd and the even moments appears also in this case.

Example 3: We match APH distributions to a distribution with Pareto tail whose pdf is given by 

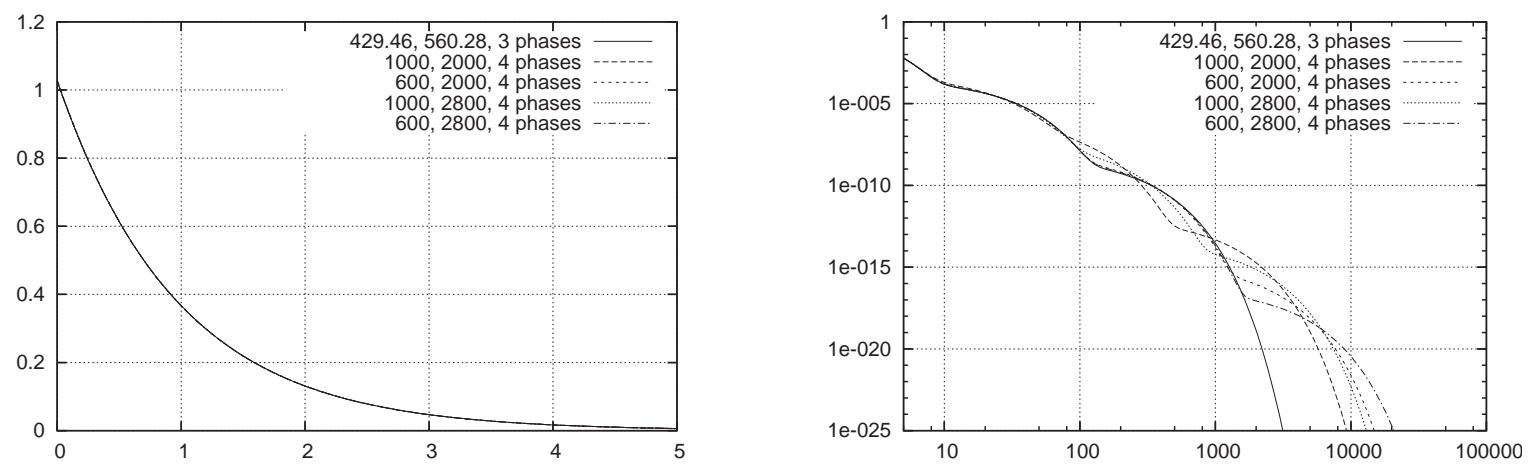

Figure 6: The body and the tail of the pdf of the distributions D6-D10

$$
f(t)= \begin{cases}\alpha B^{-1} e^{-\frac{\alpha}{B} t} & \text { for } t \leq B, \\ \alpha B^{\alpha} e^{-\alpha} t^{-(\alpha+1)} & \text { for } t>B,\end{cases}
$$

where $\alpha=3.5, B=4$ and the distribution is truncated at $10^{4}$ to ensure finite higher moments. The matching is performed based on the distributions first 3,5 or 7 moments.

The pdf and the resulting queue length distributions are depicted in Figures 8 and 9, respectively. The figures show that the approximate $\mathrm{APH}(3)$ and $\mathrm{APH}(4)$ distributions (matching 5 and 7 moments) captures the slowly decaying tail behaviour of the Pareto-like distribution.

Example 4: The normalized moments of the APH(5) distribution with

$$
\underline{p}=\left\{\frac{7}{50}, \frac{2}{25}, \frac{11}{50}, \frac{9}{25}, \frac{1}{5}\right\} \text { and } \underline{\lambda}=\left\{\frac{6}{5}, \frac{3}{2}, \frac{21}{5}, \frac{11}{2}, \frac{33}{5}\right\}
$$

\begin{tabular}{|c||c|c|c|c|c|c|}
\hline & $n_{2}$ & $n_{3}$ & $n_{4}$ & $n_{5}$ & $n_{6}$ & $n_{7}$ \\
\hline \hline D6 & 2.5 & 10 & 50 & 200 & 429.46 & 560.28 \\
D7 & 2.5 & 10 & 50 & 200 & 1000 & 2000 \\
D8 & 2.5 & 10 & 50 & 200 & 600 & 2000 \\
D9 & 2.5 & 10 & 50 & 200 & 1000 & 2800 \\
D10 & 2.5 & 10 & 50 & 200 & 600 & 2800 \\
\hline
\end{tabular}

Table 3: Normalized moments and descriptors of APH distributions of Example 2 

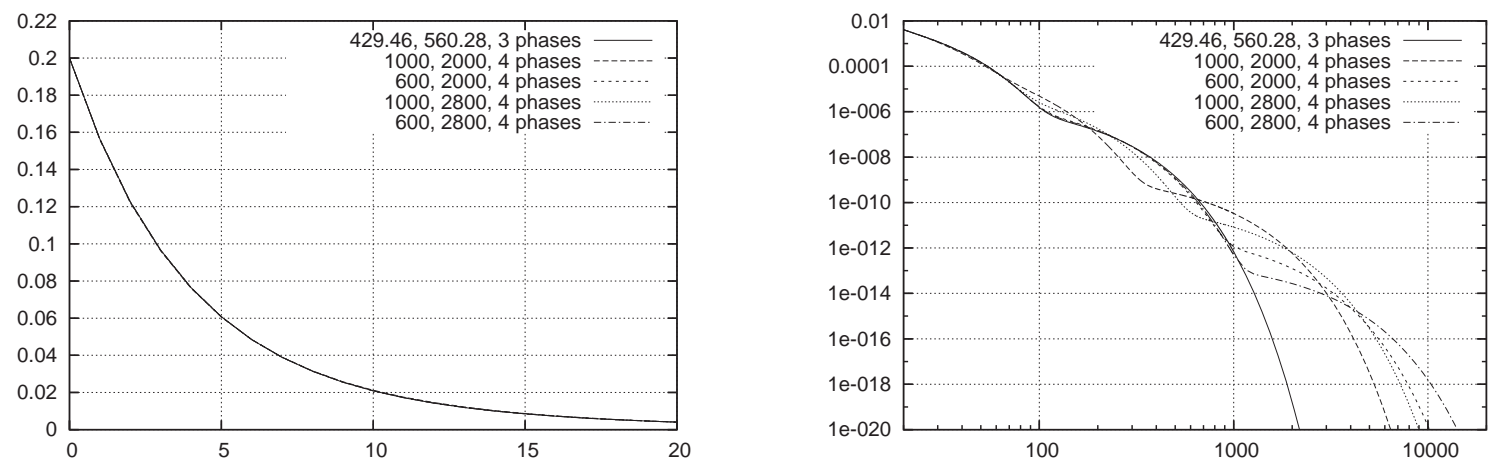

Figure 7: The body and the tail of the queue length distribution of the $\mathrm{M} / \mathrm{APH} / 1$ queue for distributions D6-D10
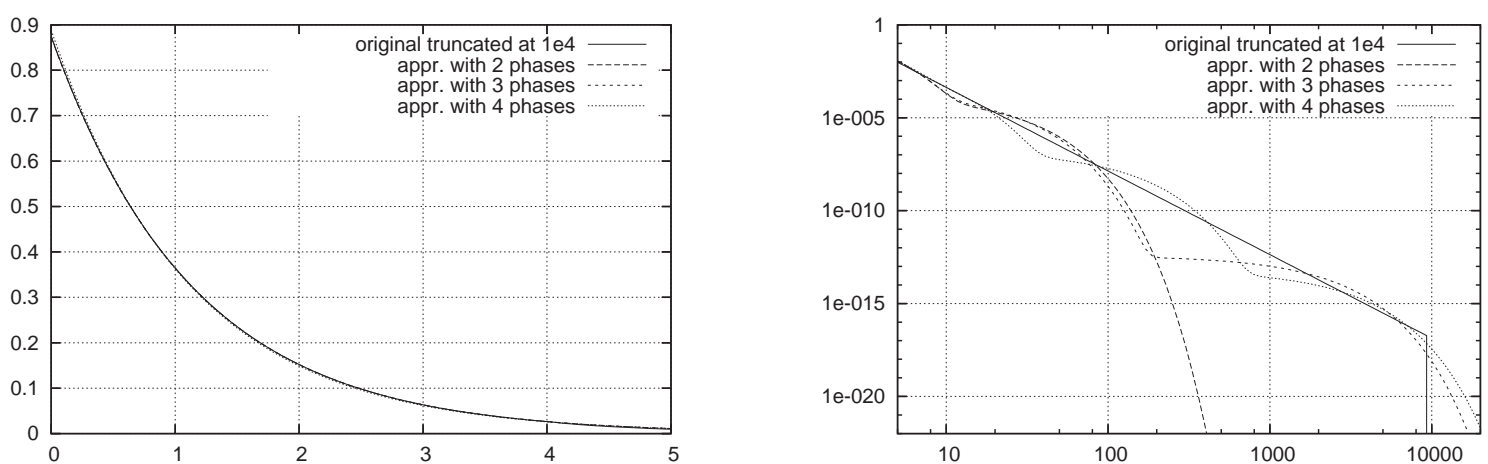

Figure 8: The pdf of the Pareto-like and the matching APH distributions

are

$$
\begin{aligned}
& n_{2}=\frac{591837100}{236021769}, \quad n_{3}=\frac{127015173550}{30307977891}, \quad n_{4}=\frac{73234144433000}{13008894074991}, \quad n_{5}=\frac{23269903941935150}{3375288482772537}, \\
& n_{6}=\frac{19293234891794731100}{2383303561732998063}, \quad n_{7}=\frac{9169582135866851564950}{988006558808808179631}, \\
& n_{8}=\frac{12639577877112590464966000}{1207476774457049490791373}, \quad n_{9}=\frac{125792074401828042303577430}{10787879718115595961848481}
\end{aligned}
$$

and its first moment is 5121/7700. If performed symbolically, i.e. the involved quantities are calculated exactly by rational fractions, the procedure returns an $\mathrm{APH}(5)$ distribution with exact rational fractions in 2.5 seconds. This distribution is the canonical representation of the original one and naturally has exactly the same moments. When performed numerically, with accuracy set to 16 effective digits (it is the normal machine precision), the procedure returns an $\mathrm{APH}(5)$ distribution whose normalized moments differ from the original normalized moments at most by $10^{-7}$. Using 32 effective digits the error disappears. 

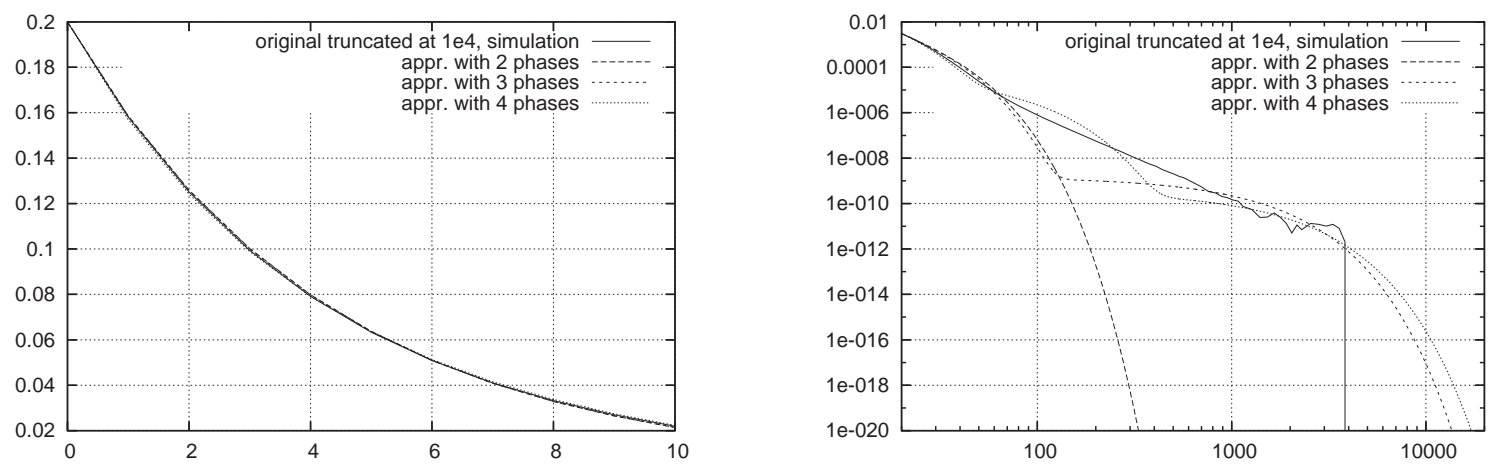

Figure 9: The queue length distribution of the $\mathrm{M} / \bullet / 1$ queue with the Pareto-like and the matching APH distributions

Example 5: The normalized moments of the $\mathrm{APH}(6)$ distribution with

$$
\underline{p}=\left\{\frac{7}{50}, \frac{2}{25}, \frac{11}{50}, \frac{9}{25}, \frac{3}{25}, \frac{2}{25}\right\} \text { and } \underline{\lambda}=\left\{\frac{6}{5}, \frac{3}{2}, \frac{16}{5}, \frac{21}{5}, \frac{11}{2}, \frac{33}{5}\right\}
$$

are

$$
\begin{aligned}
n_{2} & =\frac{13910465950}{7071632649}, \quad n_{3}=\frac{24046145958125}{7798485420889}, \quad n_{4}=\frac{4466727822468564}{1078460027763523}, \\
n_{5} & =\frac{1435435363741658131625}{281715407081136714339}, \quad n_{6}=\frac{1927035881489971461789950}{321893509448339352700643}, \\
n_{7} & =\frac{7403583591151809030412389625}{1080334855880907800908681769}, \quad n_{8}=\frac{16466099579460034164965782200920}{2134592759762499691866751133949}, \\
n_{9} & =\frac{98971954701929165646426693349164975}{11539030932796105441953896021849713}, \quad n_{10}=\frac{349390344841677602778873382799288523250}{36990438163330352563133155216939245523}, \\
n_{11} & =\frac{3638345221897932321274699737112766471711375}{352575387225254335805805592556886837427947}
\end{aligned}
$$

and its first moment is $28031 / 30800$. With symbolic computations the procedure returns an $\mathrm{APH}(6)$ distribution with exact rational fractions in 5.5 seconds. When performed numerically with low accuracy (16 or 32 effective digits) the procedure fails. With 48 effective digits the algorithm returns an $\mathrm{APH}(6)$ whose normalized moments are equal to the original normalized moments (with respect to the first 48 digits) and whose parameters differ from the parameters of the original $\mathrm{APH}(6)$ at most by $10^{-15}$.

Example 6: The normalized moments of the APH(7) distribution with

$$
\underline{p}=\left\{\frac{7}{50}, \frac{2}{25}, \frac{3}{100}, \frac{9}{25}, \frac{3}{25}, \frac{2}{25}, \frac{19}{100}\right\} \text { and } \underline{\lambda}=\left\{\frac{6}{5}, \frac{3}{2}, \frac{16}{5}, \frac{21}{5}, \frac{11}{2}, \frac{33}{5}, 7\right\}
$$




$$
\begin{aligned}
& n_{2}=\frac{231370003400}{106993755801}, \quad n_{3}=\frac{22323601720100}{6638675152819}, \quad n_{4}=\frac{6126228628514858000}{1387385281818168081}, \\
& n_{5}=\frac{5357085754374288325900}{1001941629079290768471}, \quad n_{6}=\frac{36371002969685395640867400}{5840991310566917790378547}
\end{aligned}
$$

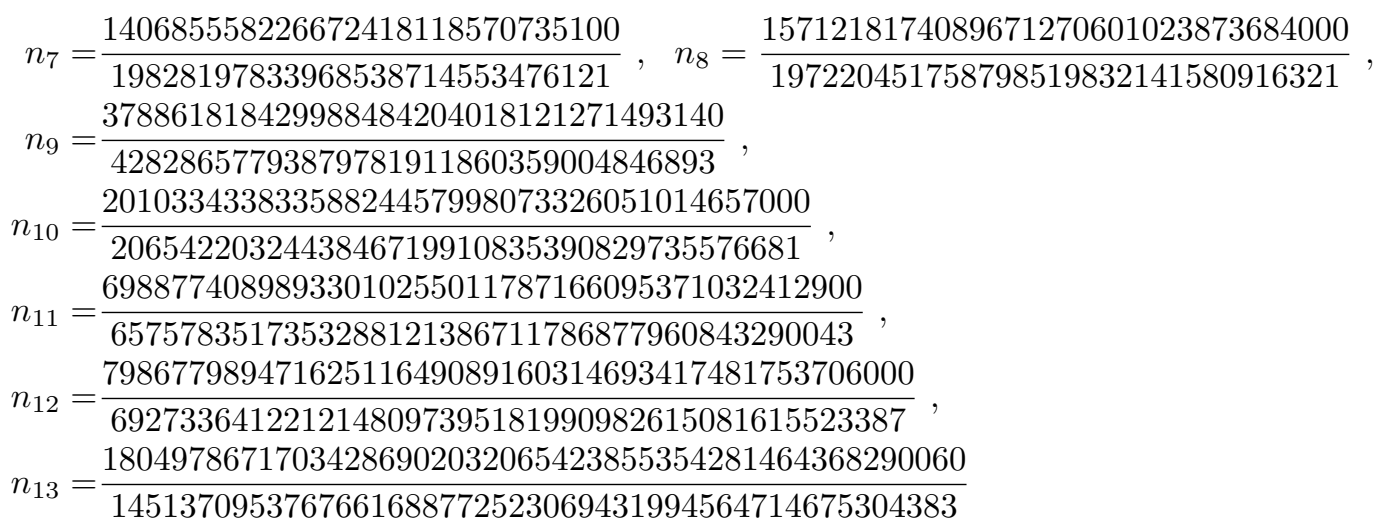

and its first moment is $109033 / 123200$. As in the previous example, with symbolic computations the procedure returns an $\mathrm{APH}(7)$ distribution with exact rational fractions in 32 seconds. With low accuracy the procedure returns incorrect values, while with higher accuracy the memory requirement of the computation is over the available amount of memory (524MB RAM).

Example 7: If the input of the procedure is the set of normalized moments of the $\mathrm{APH}(8)$ distribution with

$$
\underline{p}=\left\{\frac{7}{50}, \frac{2}{25}, \frac{3}{100}, \frac{9}{25}, \frac{3}{25}, \frac{2}{25}, \frac{11}{100}, \frac{2}{25}\right\} \quad \text { and } \underline{\lambda}=\left\{\frac{6}{5}, \frac{3}{2}, \frac{16}{5}, \frac{21}{5}, \frac{11}{2}, \frac{33}{5}, 7, \frac{36}{5}\right\}
$$

then symbolic computations returns the original $\mathrm{APH}(8)$ in 165 seconds. As in the case of the $\mathrm{APH}(7)$ distribution, numerical computations fail either for low accuracy of for too high memory requirement.

\section{Conclusion}

The paper presents a moments matching method for the $\operatorname{APH}(N)$ class. According to the authors present knowledge, this is the first procedure that provides a "Markovian" distribution based on more than 3 moments, which has practical importance in applied PH fitting.

The procedure can provide symbolic result up to order 4 and numeric results for higher orders. The 
practical applicability of the numerical procedure is limited by floating point errors and the complexity of the involved expressions. According to our experiences the procedure becomes instable around order $5-6$ with standard floating point arithmetic and requires the use of higher numerical precision.

The procedure always provides solution when the given $2 N-1$ moments are the moments of an $\operatorname{APH}(N)$ distribution (apart of numerical errors in case of numerical computation). This way the procedure allows to investigate the symbolic/numeric moment bounds of the $\operatorname{APH}(N)$ class.

\section{Acknowledgment}

The authors thanks the encouragement of the associated editor for proving Theorem 9 and 10.

\section{A Proofs of Theorem 9 and 10}

We start with a brief extract of the results presented in [10] which we use to determine the $2 N$ th and the $2 N+1$ th normalized moments of an $\mathrm{APH}(N)$ distribution in terms of the previous ones.

For a given number of phases, $N$, the following two $N+1 \times N+1$ matrices of factorial moments, $r_{i}=m_{i} / i !$, are constructed

$$
M_{2 N}=\left|\begin{array}{cccc}
r_{0} & r_{1} & \cdots & r_{N} \\
r_{1} & r_{2} & \cdots & r_{N+1} \\
\vdots & & & \vdots \\
r_{N} & r_{N+1} & \cdots & r_{2 N}
\end{array}\right|, M_{2 N+1}=\left|\begin{array}{cccc}
r_{1} & r_{2} & \cdots & r_{N+1} \\
r_{2} & r_{3} & \cdots & r_{N+2} \\
\vdots & & & \vdots \\
r_{N+1} & r_{N+2} & \cdots & r_{2 N+1}
\end{array}\right| .
$$

Then $r_{2 N}$ can be obtained as a function of $r_{i}, 0 \leq i \leq 2 N-1$ by solving the equation $\operatorname{det}\left(M_{2 N}\right)=0$. It follows from the definition of the determinant that

$$
\operatorname{det}\left(M_{2 N}\right)=\sum_{i=1}^{N+1} r_{N+i-1} \operatorname{det}_{i, N+1}\left(M_{2 N}\right)=r_{2 N} \operatorname{det}\left(M_{2 N-2}\right)+\sum_{i=1}^{N} r_{N+i-1} \operatorname{det}_{i, N+1}\left(M_{2 N}\right),
$$

where $\operatorname{det}_{i, j}\left(M_{2 N}\right)$ is the (signed) subdeterminant of the element $i, j$. From which we have

$$
r_{2 N}=-\frac{\sum_{i=1}^{N} r_{N+i-1} \operatorname{det}_{i, N+1}\left(M_{2 N}\right)}{\operatorname{det}\left(M_{2 N-2}\right)} .
$$


and, similarly, one can obtain

$$
r_{2 N+1}=-\frac{\sum_{i=1}^{N} r_{N+i} \operatorname{det}_{i, N+1}\left(M_{2 N+1}\right)}{\operatorname{det}\left(M_{2 N-1}\right)}
$$

Corollary 14. The determinant of the $n \times n$ matrix,

$$
\left|\begin{array}{cccc}
r_{a_{1}+1} & r_{a_{2}+1} & \cdots & r_{a_{n}+1} \\
r_{a_{1}+2} & r_{a_{2}+2} & \cdots & r_{a_{n}+2} \\
\vdots & & & \vdots \\
r_{a_{1}+n} & r_{a_{2}+n} & \cdots & r_{a_{n}+n}
\end{array}\right|
$$

is a sum of products $r_{i_{1}}^{p_{1}} r_{i_{2}}^{p_{2}} \cdots r_{i_{m}}^{p_{m}}$, where each products have the following property

$$
\sum_{j=1}^{m} p_{j}=n, \quad \text { and } \quad \sum_{j=1}^{m} i_{j} p_{j}=\sum_{j=1}^{n} a_{j}+\frac{n(n+1)}{2}
$$

Proof. The corollary holds for $n=1$. Assuming that the corollary holds for $k>1$ we evaluate the determinant of the matrix of (22) for $n=k+1$ using the subdeterminants of the last row and we get (23) for $k+1$.

Corollary 14 has the following consequences. The terms of $\operatorname{det}\left(M_{2 N}\right), r_{i_{1}}^{p_{1}} r_{i_{2}}^{p_{2}} \cdots r_{i_{m}}^{p_{m}}$, as well as the terms of the numerator of (20) satisfy $\sum_{j=1}^{m} p_{j}=N+1$, and $\sum_{j=1}^{m} i_{j} p_{j}=N(N+1)$. The terms of $\operatorname{det}\left(M_{2 N-2}\right)$, which give the the denominator of (20), satisfy $\sum_{j=1}^{m} p_{j}=N$, and $\sum_{j=1}^{m} i_{j} p_{j}=N(N-1)$. The terms of $\operatorname{det}\left(M_{2 N+1}\right)$ and the terms of the numerator of (21) satisfy $\sum_{j=1}^{m} p_{j}=N+1$, and $\sum_{j=1}^{m} i_{j} p_{j}=(N+1)^{2}$. The terms of $\operatorname{det}\left(M_{2 N-1}\right)$, which give the denominator of (21) satisfy $\sum_{j=1}^{m} p_{j}=N$, and $\sum_{j=1}^{m} i_{j} p_{j}=N^{2}$. As an example, for $N=2,(21)$ results in $r_{5}=\frac{-r_{3}^{3}+2 r_{2} r_{3} r_{4}-r_{1} r_{4}^{2}}{r_{2}^{2}-r_{1} r_{3}}$, where for any product in the numerator we have $\sum_{j=1}^{m} p_{j}=3$, and $\sum_{j=1}^{m} i_{j} p_{j}=9$, while for the denominator we have $\sum_{j=1}^{m} p_{j}=2$, and $\sum_{j=1}^{m} i_{j} p_{j}=4$.

From (20) and (21) one can obtain $\mathcal{F}_{2 N}\left(n_{2}, \ldots, n_{2 N-1}\right)$ and $\mathcal{F}_{2 N+1}\left(n_{2}, \ldots, n_{2 N}\right)$ using $r_{0}=1, r_{i}=$ $\frac{1}{i !} \prod_{j=1}^{i} n_{j}$, for $1 \leq i \leq 2 N-1$. Based on the above discussed properties of $r_{2 N}$ and $r_{2 N+1}$, we have the following properties of $\mathcal{F}_{2 N}\left(n_{2}, \ldots, n_{2 N-1}\right)$ and $\mathcal{F}_{2 N+1}\left(n_{2}, \ldots, n_{2 N}\right)$.

Property 1. $\mathcal{F}_{2 N}\left(n_{2}, \ldots, n_{2 N-1}\right)$ is a fraction of sums of products of normalized moments. For any product of the numerator, $n_{i_{1}}^{p_{1}} n_{i_{2}}^{p_{2}} \cdots n_{i_{n}}^{p_{n}}$, there exists a constant $K$ such that $K \leq \sum_{j=1}^{n} p_{j} \leq K+1$. For 
any product of the denominator, $n_{i_{1}}^{p_{1}} n_{i_{2}}^{p_{2}} \cdots n_{i_{n}}^{p_{n}}$, we have $K-1 \leq \sum_{j=1}^{n} p_{j} \leq K$.

As an example consider (4) in which the some of the exponents of any product of the numerator (denominator) is either two or three (one or two).

Property 2. $\mathcal{F}_{2 N+1}\left(n_{2}, \ldots, n_{2 N}\right)$ is a fraction of sums of products of normalized moments. For any product of the numerator $n_{i_{1}}^{p_{1}} n_{i_{2}}^{p_{2}} \cdots n_{i_{n}}^{p_{n}}$, there exists a constant $K$ such that $\sum_{j=1}^{n} p_{j}=K$. For any product of the denominator, $n_{i_{1}}^{p_{1}} n_{i_{2}}^{p_{2}} \cdots n_{i_{n}}^{p_{n}}$, we have $\sum_{j=1}^{n} p_{j}=K-1$.

As an example consider (5) in which the some of the exponents of any product of the numerator (denominator) is three (two).

The above two properties allows us to determine the structure of the two equations that are used to determine $a$ and $b$.

Proof of Theorem 9. By Step 1 of the procedure to construct the equation for $a$ we have

$$
n_{2 N-2}^{\prime}=\frac{(2 N-2)\left(1+b \sum_{j=1}^{2 N-2} \frac{a^{j-1}}{j !} \prod_{k=1}^{j} n_{k}\right)}{(1+b)\left(1+b \sum_{j=1}^{2 N-3} \frac{a^{j-1}}{j !} \prod_{k=1}^{j} n_{k}\right)}
$$

Then by Step 2 we obtain

$$
n_{2 N-2}^{\prime}=\frac{(2 N-2)\left(1+b \sum_{j=1}^{2 N-3} \frac{a^{j-1}}{j !} \prod_{k=1}^{j} n_{k}+\frac{b a^{2 N-3}}{(2 N-2) !} \mathcal{F}_{2 N-2}\left(n_{2}, \ldots, n_{2 N-3}\right) \prod_{k=1}^{2 N-3} n_{k}\right)}{(1+b)\left(1+b \sum_{j=1}^{2 N-3} \frac{a^{j-1}}{j !} \prod_{k=1}^{j} n_{k}\right)}
$$

According to Property $1, \mathcal{F}_{2 N-2}\left(n_{2}, \ldots, n_{2 N-3}\right)$ is of the form

$$
\frac{\sum_{i} \prod_{j=2}^{2 N-3} n_{j}^{e_{i, j}}}{\sum_{k} \prod_{l=2}^{2 N-3} n_{l}^{f_{k, l}}}
$$


and there exists a constant, $K$ such that

$$
K \leq \sum_{j=2}^{2 N-2} e_{i, j} \leq K+1, \forall i, \text { and } K-1 \leq \sum_{l=2}^{2 N-2} f_{k, l} \leq K, \forall k
$$

In Step 3, $n_{i}$ is substituted according to (15). We obtain

$$
\begin{aligned}
& n_{2 N-2}^{\prime}=(2 N-2)\left(1+b+b \sum_{j=2}^{2 N-3} \frac{a^{j-1}}{j !} \prod_{k=2}^{j} \frac{n_{k-1}^{\prime}(1+b)\left(n_{k}^{\prime}(1+b)-k\right)}{a\left(n_{k-1}^{\prime}(1+b)-(k-1)\right)}+\right. \\
& \left.\frac{b a^{2 N-3}}{(2 N-2) !} \frac{\sum_{i} \prod_{j=2}^{2 N-3}\left(\frac{n_{j-1}^{\prime}(1+b)\left(n_{j}^{\prime}(1+b)-j\right)}{a\left(n_{j-1}^{\prime}(1+b)-(j-1)\right)}\right)^{e_{i, j}}}{\sum_{k} \prod_{l=2}^{2 N-3}\left(\frac{n_{l-1}^{\prime}(1+b)\left(n_{l}^{\prime}(1+b)-l\right)}{a\left(n_{l-1}^{\prime}(1+b)-(l-1)\right)}\right)^{f_{k, l}}} \prod_{k=2}^{2 N-3} \frac{n_{k-1}^{\prime}(1+b)\left(n_{k}^{\prime}(1+b)-k\right)}{a\left(n_{k-1}^{\prime}(1+b)-(k-1)\right)}\right) / \\
& \left((1+b)\left(1+b+b \sum_{j=2}^{2 N-3} \frac{a^{j-1}}{j !} \prod_{k=2}^{j} \frac{n_{k-1}^{\prime}(1+b)\left(n_{k}^{\prime}(1+b)-k\right)}{a\left(n_{k-1}^{\prime}(1+b)-(k-1)\right)}\right)\right) .
\end{aligned}
$$

In the above equation, parts (25) and (27) do not depend on $a$. Part (26) instead depends on $a$ in such a way that the whole equation (25-27) can be rearranged to the form

$$
n_{2 N-2}^{\prime}=\frac{C_{1}+a \frac{\frac{C_{2}}{a^{K}}+\frac{C_{3}}{a^{K+1}}}{\frac{C_{4}}{a^{K-1}}+\frac{C_{5}}{a^{K}}}}{C_{6}}
$$

where the constants, $C_{i}, 1 \leq i \leq 6$ are independent of $a$. It is easy to see that (28) can be rearranged to an equation which is linear in $a$.

Proof of Theorem 10. Step 2 of the procedure to construct the equation for $b$ leads to

$$
n_{2 N-1}^{\prime}=\frac{(2 N-1)\left(1+b \sum_{j=1}^{2 N-2} \frac{a^{j-1}}{j !} \prod_{k=1}^{j} n_{k}+\frac{b a^{2 N-2}}{(2 N-1) !} \mathcal{F}_{2 N-1}\left(n_{2}, \ldots, n_{2 N-2}\right) \prod_{k=1}^{2 N-2} n_{k}\right)}{(1+b)\left(1+b \sum_{j=1}^{2 N-2} \frac{a^{j-1}}{j !} \prod_{k=1}^{j} n_{k}\right)}
$$

After application of step 3, by similar steps as in the proof of Theorem 9 and considering Property 2, we 
obtain an equation of the form

$$
\frac{D_{1}(1+b)^{2 N-2}+(1+b)^{2 N-2} \frac{\sum_{i} \frac{\prod_{j=2}^{2 N-2}\left(n_{j}^{\prime}(1+b)-j\right)^{g_{i, j}}}{D_{2, i} b^{\sum_{j=2}^{2 N-2} g_{i, j}}}}{\sum_{k} \frac{\prod_{l=2}^{2 N-3}\left(n_{l}^{\prime}(1+b)-l\right)^{h_{k, l}}}{D_{3, k} b^{\sum_{l=2}^{2 N-3} h_{k, l}}}}}{D_{4}(1+b)^{2 N-1}}
$$

where the constants, $D_{1}, D_{2, i}, D_{3, k}, D_{4}$ are independent of $b$ and $a$. Moreover

$$
\sum_{j=2}^{2 N-2} g_{i, j} \leq N, \forall i, \text { and } \sum_{l=2}^{2 N-2} h_{k, l} \leq N-1, \forall k
$$

It is easy to verify that rearranging (29) we can obtain a polynomial equation for $b$ which is of order $N$ in $b$.

\section{B Selecting the solution of interest}

To be unique the canonical representation of an acyclic phase type distributions requires that $\lambda_{1} \leq \lambda_{2} \leq$ $\ldots \leq \lambda_{N}$. The following theorem allows us to select the solution of $b$ which results in an $\operatorname{APH}(N)$ distribution in $\mathrm{CF}$, i.e., with non-decreasing intensities.

Theorem 15. Among the APH(N-1)-EXP representations of an APH(N) distribution the largest $b$ value is associated with the one where the intensity of the additional phase is the largest intensity.

Proof. Starting from an $\mathrm{APH}^{*}(N-1)$-EXP representation of an $\mathrm{APH}(N)$ distribution where the intensity of the additional phase $\left(\lambda^{*}\right)$ is not the largest intensity we apply an equivalent transformation into an $\mathrm{APH}^{\wedge}(N-1)$-EXP structure such that the intensity of the additional phase becomes the largest intensity $(\hat{\lambda})$. The steps of the transformation are depicted in Figure 10. In the first step we replace the $\operatorname{APH}^{*}(N-1)$ distribution with its CF (Fig. 10b). Consequently $\tilde{\lambda}_{N-1}=\hat{\lambda}$ is the largest intensity of the $\operatorname{APH}(N)$ distribution. The obtained structure is decomposed into an $\operatorname{APH}(N-2)$ part and two additional exponential phases (Fig. 10c). After that the last two phases are interchanged such that the $\operatorname{APH}(N)$ distribution remains the same (Fig. 10d). This transformation is due to the fact that

$$
\hat{p} \frac{\hat{\lambda}}{s+\hat{\lambda}} \frac{\lambda^{*}}{s+\lambda^{*}}+\left(1-p^{*}\right) \frac{\lambda^{*}}{s+\lambda^{*}}=\left(\hat{p}+\left(1-p^{*}\right) \frac{\hat{\lambda}-\lambda^{*}}{\hat{\lambda}}\right) \frac{\lambda^{*}}{s+\lambda^{*}} \frac{\hat{\lambda}}{s+\hat{\lambda}}+\left(1-p^{*}\right) \frac{\lambda^{*}}{\hat{\lambda}} \frac{\hat{\lambda}}{s+\hat{\lambda}} .
$$


$\mathrm{APH}(\mathrm{N})$

a)

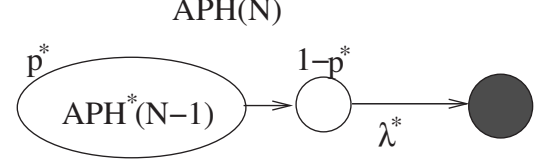

c)

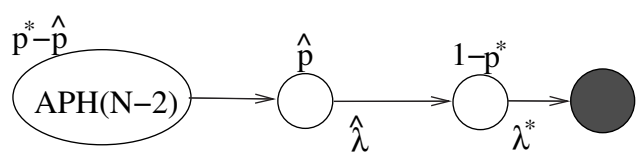

b)<smiles>[Y]C=Cc1ccccc1</smiles>

$\mathrm{APH}^{*}(\mathrm{~N}-1)$

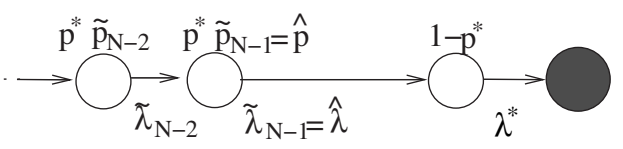

d)

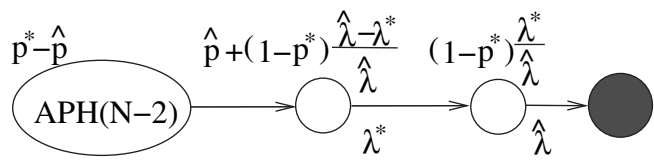

e)

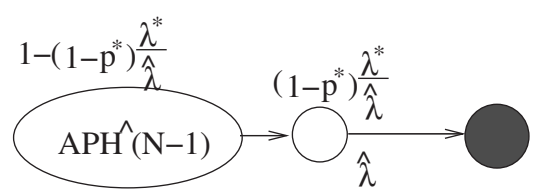

Figure 10: Equivalent transformation of $\operatorname{APH}(N-1)$-EXP forms

The $b$ parameter of the original $\mathrm{APH}^{*}(N-1)$-EXP representation, $b^{*}$ (Fig. 10a), and the final $\operatorname{APH}^{\wedge}(N-1)-$ EXP representation, $\hat{b}$ (Fig. 10e), are

$$
b^{*}=p^{*} m_{1}^{*} \lambda^{*} \text { and } \quad \hat{b}=\left(1-\left(1-p^{*}\right) \frac{\lambda^{*}}{\hat{\lambda}}\right) \hat{m}_{1} \hat{\lambda}
$$

where $m_{1}^{*}$ is the mean of $\operatorname{APH}^{*}(N-1)$ and $\hat{m}_{1}$ is the mean of $\operatorname{APH}^{\wedge}(N-1)$.

$$
\begin{gathered}
m_{1}^{*}=\sum_{i=1}^{N-1} \tilde{p}_{i} \sum_{j=i}^{N-1} \frac{1}{\tilde{\lambda}_{j}}=\left(1-\tilde{p}_{N-1}\right) m_{1}^{\prime}+\frac{1}{\tilde{\lambda}_{N-1}}=\left(1-\frac{\hat{p}}{p^{*}}\right) m_{1}^{\prime}+\frac{1}{\hat{\lambda}}, \\
\hat{m}_{1}=\frac{p^{*}-\hat{p}}{1-\left(1-p^{*}\right) \frac{\lambda^{*}}{\hat{\lambda}}} m_{1}^{\prime}+\frac{1}{\hat{\lambda}}
\end{gathered}
$$

where $m_{1}^{\prime}$ is the mean of the $\operatorname{APH}(N-2)$ and $\hat{p}=p^{*} \tilde{p}_{N-1}$. Finally substituting $m_{1}^{*}$ and $\hat{m}_{1}$ results:

$$
\begin{aligned}
\hat{b}-b^{*} & =\left(\hat{\lambda}\left(p^{*}-\hat{p}\right) m_{1}^{\prime}+1-\left(1-p^{*}\right) \frac{\lambda^{*}}{\hat{\lambda}}\right)-\left(\lambda^{*}\left(p^{*}-\hat{p}\right) m_{1}^{\prime}+p^{*} \frac{\lambda^{*}}{\hat{\lambda}}\right) \\
& =\underbrace{\left(\hat{\lambda}-\lambda^{*}\right)}_{>0} \underbrace{\left(p^{*}-\hat{p}\right)}_{p^{*}\left(1-\tilde{p}_{N-1}\right)>0} m_{1}^{\prime}+\underbrace{1-\frac{\lambda^{*}}{\hat{\lambda}}}_{>0}+\underbrace{p^{*} \frac{\lambda^{*}}{\hat{\lambda}}-p^{*} \frac{\lambda^{*}}{\hat{\lambda}}}_{0}>0 .
\end{aligned}
$$

Corollary 13 is a consequence of Theorems 11 and 15, and Corollary 12 . 


\section{References}

[1] S. Asmussen and O. Nerman. Fitting phase-type distributions via the EM algorithm. In Proceedings: "Symposium i Advent Statistik", pages 335-346, Copenhagen, 1991.

[2] A. Bobbio, A. Horváth, and M. Telek. Matching three moments with minimal acyclic phase type distributions. Stochastic Models, 21:303-326, 2005.

[3] A. Cumani. On the canonical representation of homogeneous Markov processes modelling failure-time distributions. Microelectronics and Reliability, 22:583-602, 1982.

[4] Mark Fackrell. Characterization of. Matrix-exponential distributions. PhD thesis, The University of Adelaide, School of Applied Mathematics, 2003.

[5] A. Horváth and M. Telek. PhFit: A general purpose phase type fitting tool. In Tools 2002, pages 82-91, London, England, April 2002. Springer, LNCS 2324.

[6] A. Lang and J. L. Arthur. Parameter approximation for phase-type distributions. In S. R. Chakravarty and A. S. Alfa, editors, Matrix-Analytic Methods in Stochastic Models, Lecture Notes in Pure and Applied Mathematics, pages 151-206. Marcel Dekker, Inc., 1996.

[7] T. Osogami and M. Harchol-Balter. A closed form solution for mapping general distributions to minimal PH distributions. In International Conference on Performance Tools - TOOLS 2003, pages 200-217, Urbana, IL, USA, Sept 2003. Springer, LNCS 2794.

[8] T. Osogami and M. Harchol-Balter. Necessary and sufficient conditions for representing general distributions by Coxians. In International Conference on Performance Tools - TOOLS 2003, pages 182-199, Urbana, IL, USA, Sept 2003. Springer, LNCS 2794.

[9] M. Telek and A. Heindl. Matching moments for acyclic discrete and continuous phase-type distributions of second order. International Journal of Simulation Systems, Science 83 Technology, 3(3-4):47-57, dec. 2002. Special Issue on: Analytical \& Stochastic Modelling Techniques.

[10] A. van de Liefvoort. The moment problem for continuous distributions. Technical report, University of Missouri, WP-CM-1990-02, Kansas City, 1990. 